\title{
Disease-specific structural changes in thalamus and dentatorubrothalamic tract in progressive supranuclear palsy
}

\author{
Yulia Surova $^{1,2} \cdot$ Markus Nilsson $^{3} \cdot$ Jimmy Lätt $^{4} \cdot$ Björn Lampinen $^{5}$ • Olof Lindberg ${ }^{6}$ • \\ Sara Hall ${ }^{1,2} \cdot$ Håkan Widner ${ }^{1,2}$. Christer Nilsson ${ }^{2,6}$ - Danielle van Westen ${ }^{1,4}$. \\ Oskar Hansson ${ }^{6,7}$
}

Received: 28 April 2015 / Accepted: 12 July 2015 /Published online: 8 August 2015

(C) The Author(s) 2015. This article is published with open access at Springerlink.com

\begin{abstract}
Introduction The aim of this study is to identify diseasespecific changes of the thalamus, basal ganglia, pons, and midbrain in patients with progressive supranuclear palsy (PSP), Parkinson's disease (PD), and multiple system atrophy with predominant parkinsonism (MSA-P) using diffusion tensor imaging and volumetric analysis.

Methods MRI diffusion and volumetric data were acquired in a derivation of 30 controls and 8 patients with PSP and a validation cohort comprised of controls $(n=21)$ and patients with PSP $(n=27), \operatorname{PD}(n=10)$, and MSA-P $(n=11)$. Analysis was performed using regions of interest (ROI), tract-based spatial statistic (TBSS), and tractography and results compared between diagnostic groups.

Results In the derivation cohort, we observed increased mean diffusivity (MD) in the thalamus, superior cerebellar peduncle, and the midbrain in PSP compared to controls. Furthermore,

Yulia Surova

yulia.surova@med.lu.se

1 Department of Clinical Sciences, Lund University, Lund, Sweden

2 Department of Neurology, Skåne University Hospital, 221 85 Lund, Sweden

3 Lund University Bioimaging Center, Lund University, Lund, Sweden

4 Center for Medical Imaging and Physiology, Skåne University Hospital, Lund, Sweden

5 Department of Medical Radiation Physics, Lund University, Lund, Sweden

6 Department of Clinical Sciences, Malmö, Lund University, Malmö, Sweden

7 Memory Clinic, Skåne University Hospital, Lund, Sweden
\end{abstract}

volumetric analysis showed reduced thalamic volumes in PSP. In the validation cohort, the observations of increased MD were replicated by ROI-based analysis and in the thalamus by TBSSbased analysis. Such differences were not found for patients with PD in any of the cohorts. Tractography of the dentatorubrothalamic tract (DRTT) showed increased MD in PSP patients from both cohorts compared to controls and in the validation cohort in PSP compared to PD and MSA patients. Increased MD in the thalamus and along the DRTT correlated with disease stage and motor function in PSP.

Conclusion Patients with PSP, but not PD or MSA-P, exhibit signs of structural abnormalities in the thalamus and in the DRTT. These changes are associated with disease stage and impaired motor function.

Keywords Diffusion tensor imaging · Tractography · Tract-based spatial statistics · Progressive supranuclear palsy

\section{Introduction}

Progressive supranuclear palsy syndrome (PSP) is a slowly progressing fatal neurodegenerative disease with characteristic neuropathological features including the formation of taucontaining neurofibrillary tangles and neuropil threads in the basal ganglia and brainstem structures [1-3]. According to neuropathological studies, the highest level of tau-pathology in PSP is found in the substantia nigra, globus pallidus, subthalamic nucleus, red nucleus (RN), brainstem tegmentum, and dentate nucleus [4, 5]. The thalamus often also exhibits neuronal loss and gliosis, in particular, the ventral anterior (VA) and ventral lateral (VL) thalamic nuclei [6]. PSP can often be difficult to distinguish clinically from other movement disorders, e.g., Parkinson's disease (PD), multiple 
system atrophy (MSA), and corticobasal degeneration (CBD), especially during early stages due to overlapping symptomatology. Patients with PSP exhibit motor symptoms, such as early development of postural instability, falls and rigidity and bradykinesia, as well as cognitive symptoms [3]. Fluorodeoxyglucose positron emission tomography (FDG-PET) has indicated reduced metabolism in the caudate nucleus, thalamus, and midbrain in PSP with thalamic hypometabolism correlating with impaired balance [7-9].

Diffusion tensor imaging (DTI) with estimation of parameters fractional anisotropy (FA) and mean diffusivity (MD) [10] has been used to study potential microstructural changes indicating neuronal pathway loss in the basal ganglia, midbrain, and pons in patients with PSP. Increased values of MD have been found in the thalamus, putamen, dorsal pons, and midbrain in PSP compared with controls [11-13]. Reduced values of FA have been observed in some white matter tracts, such as the superior cerebellar peduncles (SCP), corpus callosum, and inferior longitudinal fasciculus, as well as in the thalamus [14] with DTI parameters in the SCP differentiating PSP from other neurodegenerative diseases such as PD, MSA, and CBD [15-17].

Here, we aim at extending the previously reported findings of microstructural changes in subcortical structures by comparing diffusion parameter estimates from patients with PSP, MSA and PD, and controls and relating these findings to clinical symptoms. Two cohorts, with images acquired in different scanners with different protocols, were used in order to be able to study the reproducibility of the results, especially as the cohorts were quite modest in size. DTI parameters were quantified in the caudate head, putamen, and whole thalamus, as well as separately in some thalamic nuclei, the RN, SCP, deep cerebellar nuclei, pons, and midbrain, using region of interest (ROI) analysis, as well as in the dentatorubrothalamic tract (DRTT) [18] using tractography. Furthermore, tract-based spatial statistics (TBSS) analysis was employed to compare DTI parameters in the thalamus. Seemingly, we investigated whether the changes in DTI parameters were associated with severity of motor symptoms. We demonstrate specific diffusion changes in the thalamus and DRTT in patients with PSP that correlate with an increase in disease severity and worsening of motor function.

\section{Materials and methods}

\section{Subjects}

The derivation cohort included 38 subjects, 8 patients with probable PSP that were diagnosed according to the National Institute of Neurological Disorders and Stroke (NINDS) criteria [3], and 30 healthy age- and sex-matched controls. Healthy controls had no previous neurologic or psychiatric diseases. Adjunctive inclusion criteria for patients with PSP were a poor or absent response to levodopa. All patients were recruited at Skåne University Hospital. Patients and controls were evaluated using clinical assessments, among these, the Hoehn and Yahr staging scale (H\&Y) [19], the Schwab and England activities of daily living scale (S\&E) [20], as well as the Unified Parkinson's Disease Rating Scale (UPDRS)-3 [21] on the on stage. The tandem gait test [22] was included to assess disturbances in balance. Cognitive function was assessed by Mini Mental State Examinations (MMSE) [23] and The Quick Test of Cognitive Speed (AQT) [24]. In addition, the PSP rating scale (PSPRS) was administered to PSP patients [25]. Patients were evaluated three times at typical intervals of 2 years, but here, we report on the PSPRS score closest to the MRI scan date (Table 1). The PSPRS comprises 28 items in six areas, the History and daily activities, the Mentation, the Bulbar, the Ocular motor, the Limb motor, and the Gate and midline with the total score ranging from 0 (normal) to 100. There was no attempt to evaluate patients off medication.

In the validation cohort, participants were recruited from the Neurology and Memory Clinics at Skåne University Hospital, Sweden, between 2008 and 2011. For the present work, 69 subjects were included, with a clinical diagnosis of probable PD $(n=10)$, probable PSP $(n=27)$, or probable MSA-P (parkinsonian variant of MSA) $(n=11)$. In addition, neurologically healthy controls were recruited $(n=21)$. Clinical diagnosis was assessed by neurologists experienced in parkinsonian disorders according to NINDS criteria $[3,26$, 27]. Postmortem diagnoses were available from one PSP and one MSA-P cases. Controls, age- and sex-matched, were recruited from the Swedish population registry and had no previous neurologic or psychiatric diseases. The H\&Y [19] and the $S \& E[20]$ were assessed retrospectively from medical records, without any information about on/off state during visit to doctor.

Since vascular lesions could mimic parkinsonism or be subclinical in healthy subjects, patients with anatomic MRI abnormalities and vascular lesions in midbrain and basal ganglia were ruled out by an experienced neuroradiologist who evaluated MRI scans for each subject in both cohorts.

\section{Data acquisition and processing}

In the derivation cohort, imaging was performed using a $3 \mathrm{~T}$ Siemens Skyra MR scanner equipped with a 20 channel head coil. The DTI data were collected using a single-shot EPI (TE/ TR $70 / 7500 \mathrm{~ms} / \mathrm{ms}$ ) sequence with diffusion encoding in 30 directions using $b$ values of 0 and $1000 \mathrm{~s} / \mathrm{mm}^{2}$, IPAT factor of 2 , voxel size of $2 \times 2 \times 2 \mathrm{~mm}^{3}$, with an acquisition time $4 \mathrm{~min}$ $15 \mathrm{~s}$. In the validation cohort, imaging was performed 


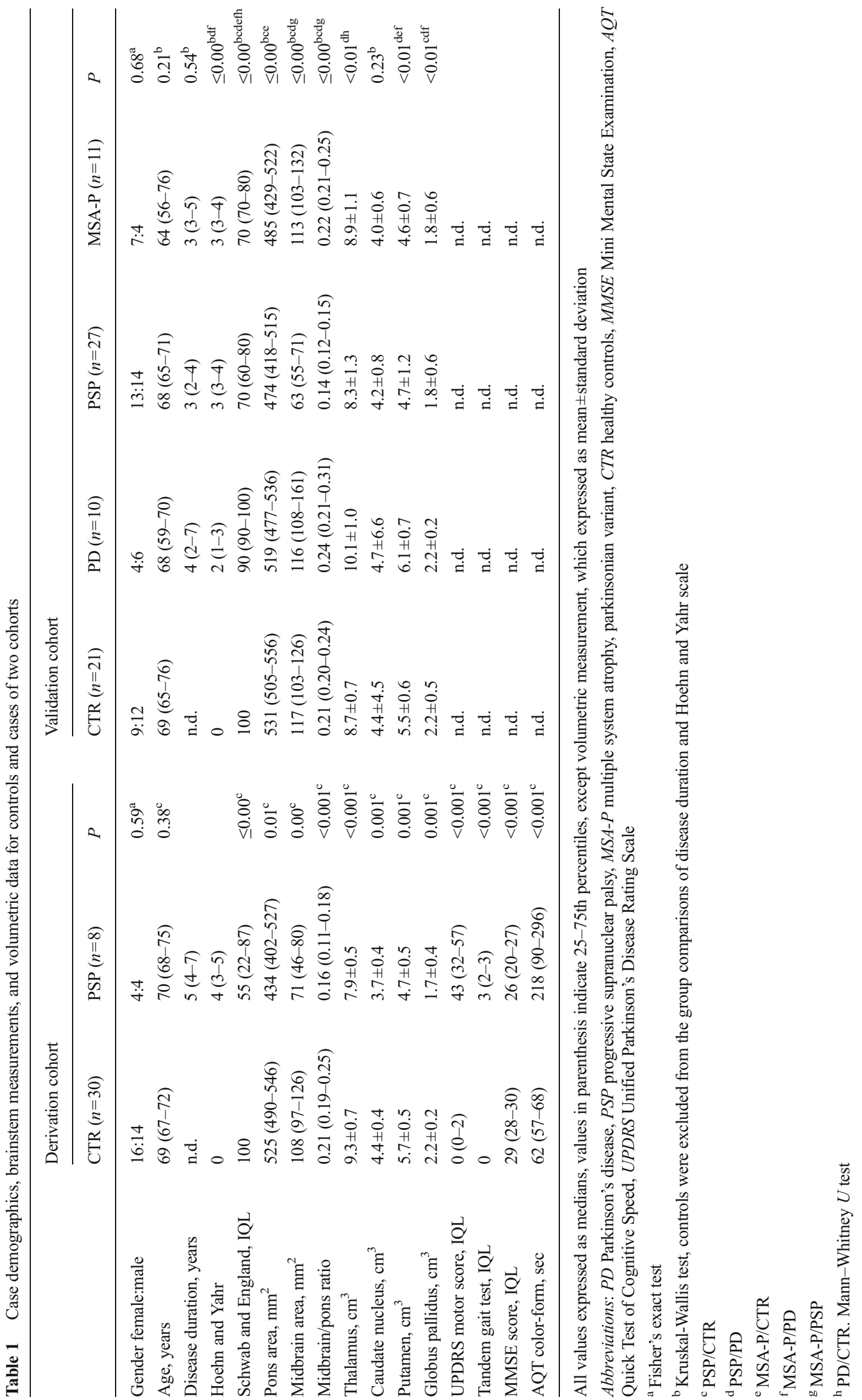


using a 3 T Philips Achieva MR scanner, equipped with an eight-channel head coil. DTI data were collected using a single-shot EPI sequence (TE/TR 90/7840 ms/ $\mathrm{ms}$ ) with diffusion encoding in 48 directions, $b$ values of 0 and $800 \mathrm{~s} / \mathrm{mm}^{2}$, and SENSE factor of 2.5; 60 slices were acquired with voxel size $2 \times 2 \times 2 \mathrm{~mm}^{3}$. The lower $b$ value in the validation cohort was used to reduce the acquisition time. However, this will result in slightly higher values for MD [28] and thus values for MD from the derivation and validation cohorts are expected to differ. Subject motion and eddy-current correction was performed using ElastiX [29], and parameter maps were calculated using in-house developed software. For tractography, data was processed using MRtrix (Brain Research Institute, Melbourne, Australia, http://www.brain.org.au/software/) [30], including constrained spherical deconvolution (CSD) to model multiple fiber orientations in each voxel.

Volumetric data were acquired in the derivation cohort using a MPRAGE sequence with TR/TE $7 / 3 \mathrm{~ms}$, flip angle $9^{\circ}$, resolution $1 \times 1 \times 1 \mathrm{~mm}^{3}$ and in validating cohort using a T1-weighted TFE sequence with TR/TE $8 / 4 \mathrm{~ms}$, flip angle $10^{\circ}$, resolution $1 \times 1 \times 1 \mathrm{~mm}^{3}$.

\section{Analysis of diffusion parameters}

a) ROI-based analysis

For ROI-based estimation of diffusion parameters, ROIs were outlined manually on parameter maps by one trained investigator (YS). All ROIs were outlined twice with an interval of 3 months (average intra-rater variability $>0.9$ for all ROIs). FA- and directionally color-encoded FA maps were used to outline all ROIs, except for the RN and deep cerebellar nuclei (DCN), where the non-diffusion-weighted map was used (Fig. 1). The ROI size was adjusted in each subject to maximize coverage of each structure, while minimizing partial volume effects from neighboring areas. Contamination from cerebrospinal fluid (CSF), which has isotropic diffusion with a high MD, was avoided by excluding voxels adjacent to the third and lateral ventricles.

The head of the caudate nucleus was delineated in a single slice at the level where it was most conspicuous. The thalamus and putamen were delineated in 5-8 consecutive slices at the level of the internal capsule, the thalamus adjacent to the interthalamic adhesion, and the putamen to the extreme capsule. The VA and VL nuclei of the thalamus (VAVL) were identified on FA color maps as green voxels (Fig. 1) $[31,32]$, in the anterior part of the lateral thalamus close to the genu of the internal capsule, while excluding the three most medial voxels that were regarded to comprise the medial dorsal nuclei. The lateral posterior nucleus (LP) and ventral posterior (VP) nuclear complex of the thalamus (LPVP) were identified on FA color maps as violet voxels (Fig. 1) $[31,32]$ adjacent to the posterior limb of the internal capsule and anterior to the pulvinar in red. The RN was identified as a circular area of signal hypointensity in the midbrain on non-diffusionweighted maps and delineated in at least two consecutive axial slices. The SCP was delineated on two consecutive sagittal slices. The midbrain and the pons were delineated on five consecutive sagittal

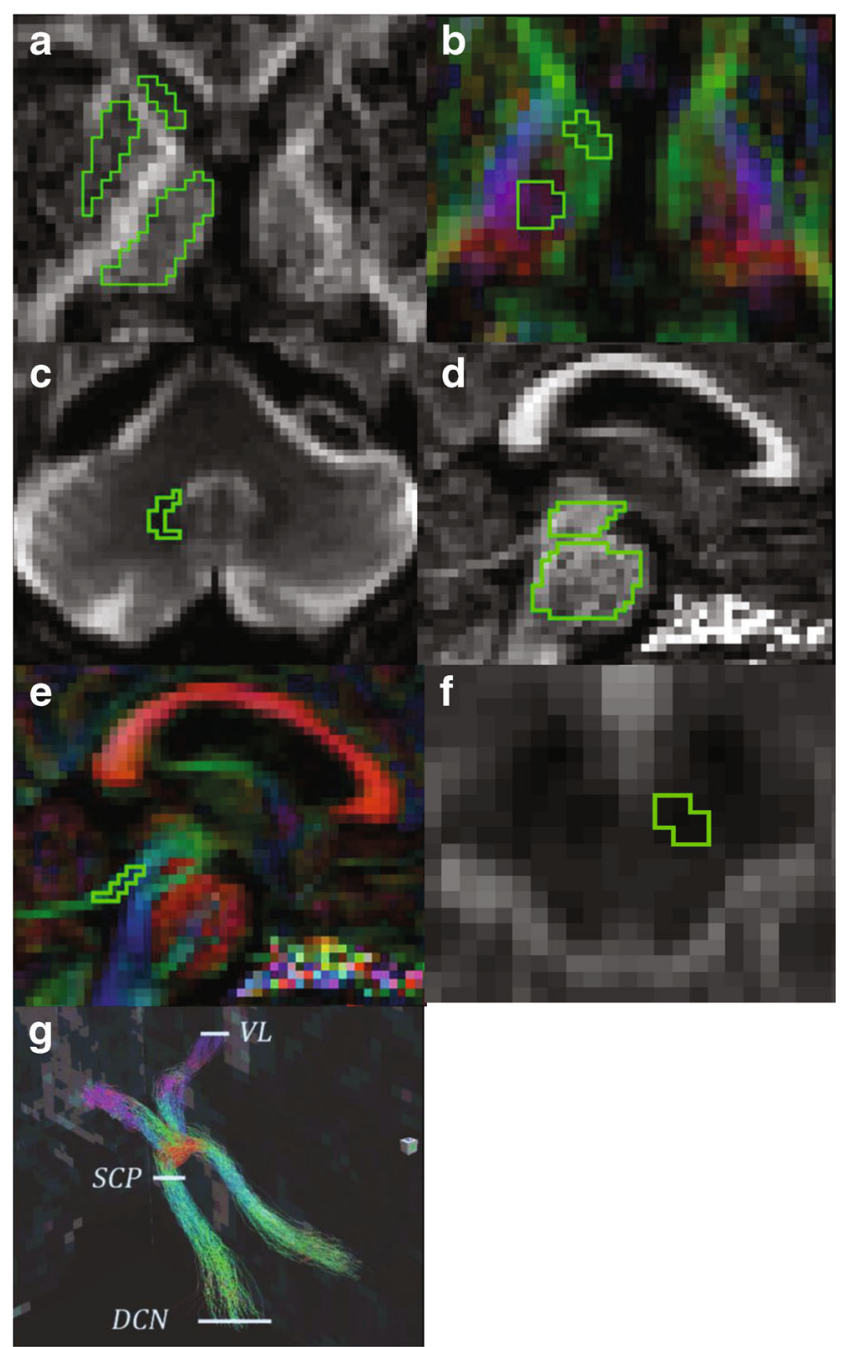

Fig. 1 Region of interests in subcortical structures and the superior cerebellar peduncle and tractography of the dentatorubrothalamic tract. Region of interests (ROIs) are placed in a the putamen, caudate head, thalamus; $\mathbf{b}$ the ventral anterior and ventral lateral nuclei of the thalamus (VL), green colored voxels and the lateral posterior nucleus and ventral posterior nuclear complex of the thalamus, violet colored voxels; $\mathbf{c}$ the red nucleus; $\mathbf{d}$ the pons and midbrain; e superior cerebellar peduncle (SCP); $\mathbf{f}$ deep cerebellar nuclei (DCN); $g$ the left and right dentatorubrothalamic tract defined by ROIs were placed in DCN, SCP, and contralateral VL 


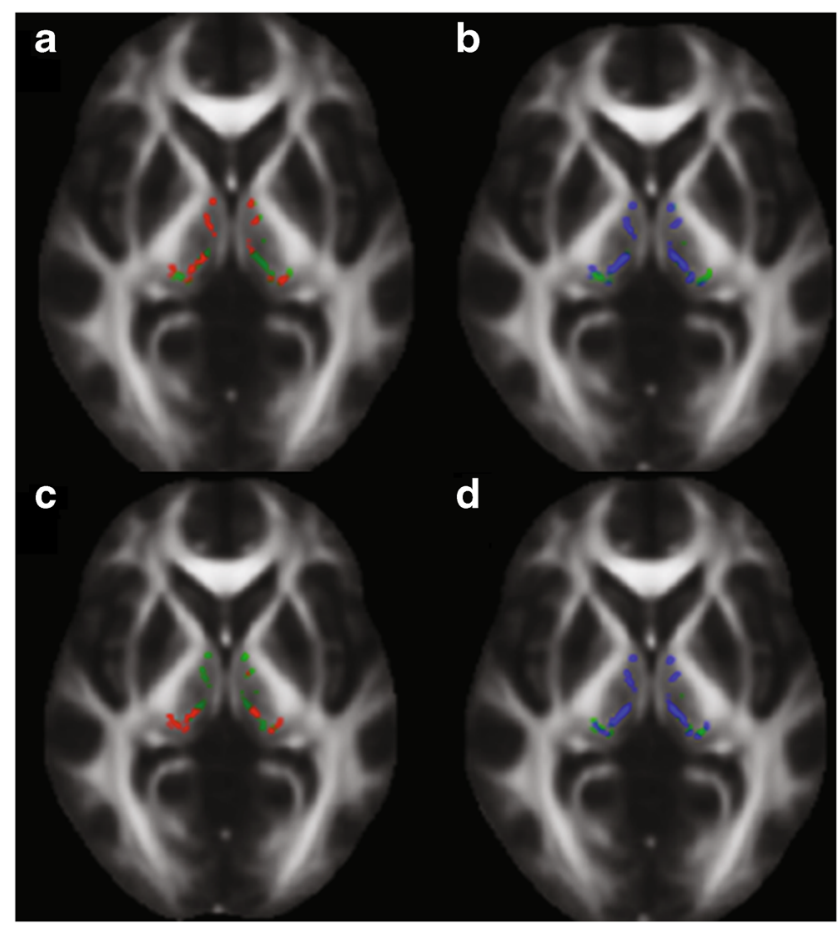

Fig. 2 Areas in thalamus with significantly decreased fractional anisotropy and increased mean diffusivity in patients with progressive supranuclear palsy when compared to healthy controls. Results of the tract-based spatial statistics (TBSS) analysis in the thalamus, showing regions of significant decreased fractional anisotropy (red voxels) and increased mean diffusivity (blue voxels) in patients with progressive supranuclear palsy when compared to healthy controls. $\mathbf{a}$ and $\mathbf{b}$ correspond to the derivation cohort, while $\mathbf{c}$ and $\mathbf{d}$ correspond to the validation cohort. Green voxels are voxels on the TBSS-skeleton where no significance was found

slices, with ROIs including the whole structure. The manual approach proposed by Oba et al. [33] was used to identify the boundaries of the pons and the midbrain. For each structure, the average FA and MD value from the right and left hemisphere was calculated.

b) tract-based spatial statistics analysis

TBSS (v 1.03), part of the FRMIB Software Library (FSL), was employed as a complementary analysis tool for diffusion parameters in the thalamus. Comparisons were performed in PSP vs controls in the derivation cohort and in PSP vs controls, PD and MSA-P in the validation cohort (Fig. 2) [34]. FA and MD maps were registered onto the $1 \mathrm{~mm}^{3}$ FMRIB58 FA template in MNI152 standard space, using the linear and nonlinear registration tools FLIRT and FNIRT $[35,36]$. Before registration, the diffusion maps were masked with the FSL Brain Extraction Tool (BET) [37]. The normalized maps were skeletonized by projection onto the FMRIB58 template skeleton. Finally, the skeletonized maps were masked to include only voxels from the thalamus. The masking was done using the left and right thalamus regions in the MNI152 space Harvard-Oxford subcortical atlas, together with the requirement that the MD of the normalized maps must be less than unity in the control subjects of the derivation cohort [38].

c) Tractography of the DRTT

For probabilistic tractography of the DRTT, its inferior and superior part were constructed each using a seed ROI placed in the DCN and VAVL of the contralateral thalamus, respectively, and an include ROI in the SCP (Fig. 1). The left and right DRTT were then constructed by combining the inferior and superior DRTT into one tract, selecting fibers passing through include ROIs in these three locations, DCN, SCP, and VAVL. All tracts were visually inspected. The tractography procedure did not generate tracts in two controls from the derivation cohort (unilaterally) as well as in four PSP patients (one patient bilaterally and three unilateral) and one control (unilateral) in the validation cohort.

\section{Volumetric analysis}

The diameter and area of the midbrain and pons, the midsagittal slice were assessed according to Oba [33]. Thus, measurements included the antero-posterior diameter of the midbrain (AP-diameter), the distance between the interpeduncular fossa, and the aqueduct in the midbrain proper without the quadrigeminal plate (IF-AQ-diameter), the surface of the pons (P-area), and the surface of the midbrain (M-area) [33, 39]. The $\mathrm{P} / \mathrm{M}$ ratio was calculated as the ratio of the $\mathrm{P}$-area to the $\mathrm{M}$-area. Measurements were performed twice and median values were used.

Automated volumetric measurement was performed with FIRST [40]. Correction for intracranial volume (ICV) was achieved by multiplying each volume with the scaling factor that estimates the scaling between the subject's image and standard space and that is provided by the sienax toolbox [41]. The mean volume of the right and left thalamus, caudate nucleus, putamen, and globus pallidus were used.

\section{Statistical analysis}

Statistical analysis was performed with SPSS Statistics 20 for Windows (IBM Corporation, Somers, NY, USA). Within each cohort, differences between groups in demographic and clinical categorical variables were analyzed using the Fisher's exact test for dichotomized data and Kruskal-Wallis test 
followed by Mann-Whitney $U$ test for continuous data. Correlation between diffusion parameters and clinical scores was tested using Spearman correlation (Spearman's Rho). The level of significance was set to $P<0.05$ in the derivation and $P<0.008$ in the validation cohorts. An adjustment for multiple comparisons between the 4 control/patient categories (i.e., 6 comparisons) was made in the validation cohort, leading to an adjusted significance level of $P=0.008$ using Bonferroni correction. Correlation between diffusion parameters and PSPRS items was tested with linear logistic regression. Adjustment for age was performed by binary logistic regression analysis with diffusion parameter values that were significantly different between groups, based on the results of Mann-Whitney $U$ test. For comparison between PSP and controls, effect sizes were computed in the form of differences in diffusional parameters group means.

To study the ability of DTI and DTT measurements to distinguish PSP from controls, MSA-P and PD in the validation cohort, receiver operator characteristic curve analysis (ROC) was performed.

Statistical processing of TBSS data was performed using Threshold-Free Cluster Enhancement of FSL Randomize (v 2.9), with 7500 permutations for the null distribution [42]. Finally, for each comparison and diffusion parameter, we computed effect sizes in the form of difference in group means in the skeletonized space in the thalamus region.

\section{Results}

\section{Demographics}

Demographic clinical data of patients and controls in both cohorts are reported in Table 1. Study participants of the derivation cohort were more extensively characterized compared to the validation cohort. Age, gender, and disease duration were similar in the PD, MSA-P, and PSP groups in both cohorts; however, patients with PSP and MSA-P were more disabled compared to controls as well as patients with PD.

\section{Diffusion parameters}

a) Region of interests based analysis

In the derivation cohort, we found that patients with PSP have increased MD in the whole thalamus, the thalamic nuclei (VAVL and LPVP), and the midbrain (Table 2). Significant changes of MD (increase with 9-12\%) were found in the caudate head, thalamus, VAVL, LPVP, and midbrain. Values of FA were reduced with $12-19 \%$ for the SCP and midbrain in PSP patients.

In the validation cohort, patients with PSP showed higher MD values in the whole thalamus, the VAVL nuclei of the thalamus and the midbrain compared to controls, patients with PD, and patients with MSA-P (Table 2). Patients with MSA-P displayed higher MD values in the pons than controls and patients with $\mathrm{PD}$ but not compared to patients with PSP (Table 2). The values of MD in PSP were increased with 6-9\% in the putamen, thalamus, VAVL, LPVP, and pons and with $12-15 \%$ for midbrain, SCP, and red nucleus. Values of FA in PSP were reduced with 5-6\% in the putamen and VAVL, with $11-15 \%$ in midbrain and SCP.

In the patients with PSP, MD changes in the thalamus did not correlate with volumetric measurements in any of the cohorts.

b) tract-based spatial statistics analysis

In the derivation cohort, PSP patients showed significantly higher MD than controls in $75 \%$ of the skeletonized voxels in the thalamus (Fig. 2). In the validation cohort, patients with PSP were found to have a higher MD in the thalamus than controls (75\% significant voxels). In the derivation cohort, a higher MD was also found in the PSP group compared to both PD (67 \% significant voxels) and MSA-P (53\% significant voxels). Furthermore, a reduced FA was observed in PSP vs controls in both cohorts and in PSP VS IPD in the derivation cohort (30-50 \% significant voxels).

\section{c) Tractography of the DRTT}

As many of the changes in diffusion parameters in patients with PSP observed above were localized in structures associated with the DRTT (i.e., SCP, midbrain, and ventral thalamus); probabilistic tractography of this tract was performed. In the derivation cohort, elevation in MD and reduction of FA was seen in patients with PSP, even though it only reached significance on the right side $(P<0.05)$ (Table 2 and Fig. 3c, d). Value of FA was reduced with $19 \%$, and value of MD was increased with $14 \%$ for right DRTT in PSP patients. Similar to the derivation cohort, tractography of the DRTT in patients with PSP in the validation cohort exhibited reduced FA and increased MD in the DRTT on both sides when compared to both controls and patients with PD or MSA-P (Table 2 and Fig. 3a, b). The values of FA were reduced with $21-28 \%$, and values 
Table 2 Regional fractional anisotropy and mean diffusivity values in two cohorts

\begin{tabular}{|c|c|c|c|c|c|c|c|}
\hline \multirow[t]{2}{*}{ Region } & \multirow{2}{*}{$\begin{array}{l}\text { Parameter } \\
\text { FA }\end{array}$} & \multicolumn{2}{|l|}{ Derivation cohort } & \multicolumn{4}{|l|}{ Validation cohort } \\
\hline & & CTR & PSP & CTR & PD & PSP & MSA-P \\
\hline Caudate head & & $0.16(0.14-0.18)$ & $0.18(0.15-0.20)$ & $0.16(0.14-0.20)$ & $0.17(0.14-0.19)$ & $0.18(0.15-0.20)$ & $0.16(0.15-0.21)$ \\
\hline Putamen & & $0.15(0.14-0.17)$ & $0.16(0.13-0.19)$ & $0.19(0.17-0.20)$ & $0.20(0.18-0.22)$ & $0.18(0.17-0.20)^{\mathrm{c}}$ & $0.22(0.20-0.24)$ \\
\hline Thalamus & & $0.29(0.28-0.31)$ & $0.28(0.25-0.30)$ & $0.32(0.30-0.33)$ & $0.31(0.30-0.33)$ & $0.30(0.29-0.33)$ & $0.31(0.29-0.33)$ \\
\hline VAVL & & $0.28(0.26-0.30)$ & $0.27(0.24-0.30)$ & $0.31(0.30-0.33)$ & $0.32(0.29-0.34)$ & $0.29(0.26-0.32)^{\mathrm{a}}$ & $0.31(0.29-0.33)$ \\
\hline LPVP & & $0.27(0.25-0.29)$ & $0.26(0.24-0.28)$ & $0.29(0.26-0.32)$ & $0.31(0.29-0.33)$ & $0.31(0.29-0.33)$ & $0.31(0.29-0.35)$ \\
\hline Red nucleus & & $0.45(0.40-0.52)$ & $0.45(0.40-0.51)$ & $0.54(0.49-0.58)$ & $0.53(0.47-0.54)$ & $0.50(0.45-0.56)$ & $0.51(0.43-0.56)$ \\
\hline SCP & & $0.66(0.62-0.68)$ & $0.54(0.42-0.70)^{\mathrm{a}}$ & $0.78(0.75-0.82)$ & $0.79(0.71-0.84)$ & $0.66(0.60-0.71)^{\mathrm{bc}}$ & $0.75(0.73-0.76)$ \\
\hline Pons & & $0.35(0.34-0.38)$ & $0.33(0.32-0.36)$ & $0.41(0.39-0.46)$ & $0.40(0.37-0.44)$ & $0.39(0.36-0.42)$ & $0.39(0.34-0.39)$ \\
\hline Midbrain & & $0.40(0.36-0.42)$ & $0.35(0.30-0.38)^{\mathrm{a}}$ & $0.45(0.43-0.48)$ & $0.47(0.45-0.48)$ & $0.40(0.38-0.43)^{\mathrm{abc}}$ & $0.45(0.43-0.49)$ \\
\hline DRTT left & & $0.38(0.33-0.39)$ & $0.33(0.25-0.38)$ & $0.38(0.35-0.40)$ & $0.39(0.36-0.41)$ & $0.30(0.27-0.34)^{\mathrm{abc}}$ & $0.37(0.31-0.38)$ \\
\hline \multirow[t]{2}{*}{ DRTT right } & & $0.37(0.33-0.40)$ & $0.30(0.26-0.35)^{\mathrm{a}}$ & $0.36(0.33-0.37)$ & $0.38(0.36-0.44)$ & $0.26(0.24-0.29)^{\mathrm{abc}}$ & $0.32(0.31-0.36)^{\mathrm{c}}$ \\
\hline & MD & & & & & & \\
\hline Caudate head & & $0.68(0.66-0.73)$ & $0.74(0.63-0.79)^{\mathrm{a}}$ & $0.79(0.76-0.87)$ & $0.81(0.77-0.91)$ & $0.82(0.75-0.96)$ & $0.76(0.68-0.85)$ \\
\hline Putamen & & $0.68(0.64-0.71)$ & $0.70(0.66-0.76)$ & $0.81(0.75-0.86)$ & $0.78(0.76-0.86)$ & $0.86(0.80-0.96)^{\mathrm{a}}$ & $0.88(0.84-1.04)^{\mathrm{ed}}$ \\
\hline Thalamus & & $0.71(0.69-0.73)$ & $0.79(0.71-0.89)^{\mathrm{a}}$ & $0.79(0.75-0.82)$ & $0.77(0.76-0.81)$ & $0.84(0.79-0.88)^{\mathrm{abc}}$ & $0.78(0.77-0.80)$ \\
\hline VAVL & & $0.72(0.70-0.74)$ & $0.81(0.71-0.89)^{\mathrm{a}}$ & $0.79(0.76-0.81)$ & $0.79(0.77-0.83)$ & $0.86(0.82-0.93)^{\mathrm{abc}}$ & $0.80(0.76-0.83)$ \\
\hline LPVP & & $0.70(0.68-0.72)$ & $0.77(0.71-0.93)^{\mathrm{a}}$ & $0.76(0.72-0.81)$ & $0.71(0.70-0.75)$ & $0.81(0.76-0.89)^{\mathrm{bc}}$ & $0.74(0.74-0.75)$ \\
\hline Red nucleus & & $0.48(0.43-0.53)$ & $0.55(0.42-0.59)$ & $0.53(0.45-0.60)$ & $0.530 .48-0.58)$ & $0.61(0.55-0.68)^{\mathrm{a}}$ & $0.61(0.52-0.62)$ \\
\hline $\mathrm{SCP}$ & & $0.82(0.76-0.86)$ & $0.85(0.79-1.11)$ & $0.83(0.76-0.88)$ & $0.82(0.76-0.89)$ & $0.94(0.90-1.10)^{\mathrm{ab}}$ & $0.92(0.87-0.97)^{\mathrm{d}}$ \\
\hline Pons & & $0.63(0.60-0.66)$ & $0.65(0.62-0.70)$ & $0.72(0.70-0.75)$ & $0.72(0.70-0.75)$ & $0.76(0.72-0.79)^{\mathrm{a}}$ & $0.75(0.75-0.78)^{\mathrm{de}}$ \\
\hline Midbrain & & $0.68(0.66-0.72)$ & $0.74(0.71-0.81)^{\mathrm{a}}$ & $0.74(0.73-0.76)$ & $0.73(0.71-0.76)$ & $0.83(0.80-0.85)^{\mathrm{abc}}$ & $0.75(0.70-0.78)$ \\
\hline DRTT left & & $0.89(0.84-0.93)$ & $1.02(0.86-1.18)$ & $1.1(1.02-1.15)$ & $1.03(0.97-1.11)$ & $1.37(1.27-1.52)^{\mathrm{abc}}$ & $1.21(1.02-1.23)^{\mathrm{c}}$ \\
\hline DRTT right & & $0.87(0.80-0.93)$ & $0.99(0.82-1.07)^{\mathrm{a}}$ & $1.16(1.05-1.20)$ & $1.06(0.99-1.10)$ & $1.50(1.34-1.55)^{\mathrm{abc}}$ & $1.22(1.16-1.30)^{\mathrm{c}}$ \\
\hline
\end{tabular}

$\mathrm{MD}\left(10^{\wedge}-3 \mathrm{~mm}^{\wedge} 2 / \mathrm{s}\right)$. All values expressed as medians, values in parenthesis indicate $25-75$ th percentiles. FA values in caudate head and putamen are low and should therefore be interpreted with caution

Abbreviations: FA fractional anisotropy, $M D$ mean diffusivity, $V A V L$ ventral anterior and ventral lateral nuclei of the thalamus, $L P V P$ lateral posterior nucleus and ventral posterior nuclear complex of the thalamus, $S C P$ superior cerebellar peduncle, $D R T T$ dentatorubrothalamic tract, $P D$ Parkinson's disease, $P S P$ progressive supranuclear palsy, MSA-P multiple system atrophy, parkinsonian variant, $C T R$ healthy control

${ }^{\mathrm{a}} \mathrm{PSP} / \mathrm{CTR}$

${ }^{\mathrm{b}} \mathrm{PD} / \mathrm{PSP}$

${ }^{c}$ PSP/MSA-P

${ }^{\mathrm{d}}$ MSA-P/CTR

${ }^{\mathrm{e}}$ MSA-P/PD. Mann-Whitney $U$ test

of MD in PSP were increased with 24-29\% in the left and right DRTT.

\section{Volumetric measurements}

The volumes of the thalamus, the putamen, and the globus pallidus were reduced in patients with PSP in both cohorts (Table 1). The midbrain area was reduced in patients with PSP when compared to PD, MSA-P, and control subjects (Table 1). In patients with MSA-P (validation cohort only), the volumes of the putamen and the globus pallidus were reduced (Table 1). Only the thalamic volume and the midbrain area were specifically reduced in PSP.

\section{Correlation between clinical scales and diffusion parameters of thalamus and dentatorubrothalamic tract in progressive supranuclear palsy}

In the derivation cohort, increased MD in the whole thalamus, VAVL, and LPVP correlated with increased disease stage $(\mathrm{H} \& \mathrm{Y})$ and with reduced rating scores of activities of daily living (S\&E) (Spearman's Rho $=-0.732-0.756, P<0.05$ ). Very similar findings were obtained in the validation cohort. There was a negative correlation between MD and disease 
stage (H\&Y) in PSP patients in the validation cohort, similar to that in the derivation cohort, for values obtained in the whole thalamus and in the VAVL nuclei (Spearman's Rho= 0.456 and $0.431, P=0.017$ and $P=0.025$, respectively). Further, higher MD in the LPVP correlated negatively with functional measures of activity of daily living (S\&E) (Spearman's Rho $=-0.467$ and $-0.489, P=0.014$ and $P=$ 0.010 , respectively).

a

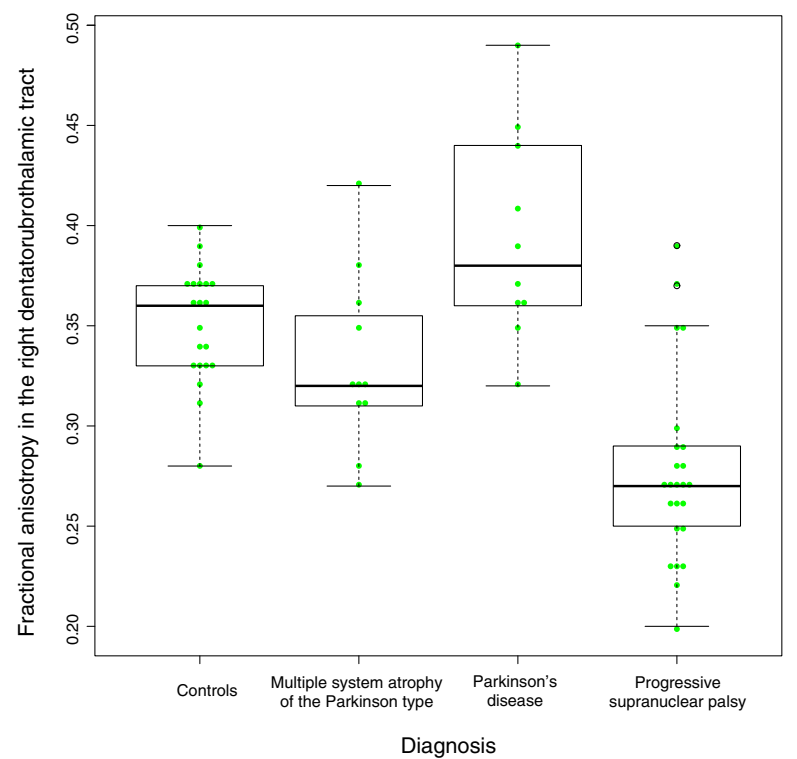

C

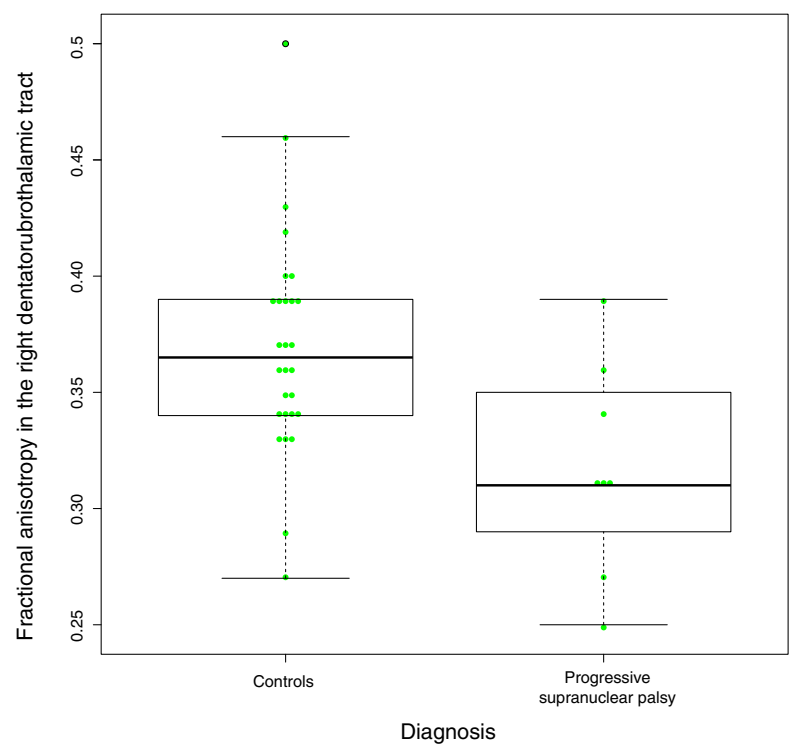

Fig. 3 Fractional anisotropy and mean diffusivity in dentatorubrothalamic tract in derivation and validation cohorts. a Fractional anisotropy values in the right dentatorubrothalamic tract validation cohort. b Mean diffusivity values in the right dentatorubrothalamic tract validation cohort, $\left[10^{\wedge}-\right.$
The thalamic volume correlated with neither S\&E nor H\&Y (both cohorts).

In the derivation cohort, the patients with PSP were also assessed with other clinical rating scales including UPDRS and the PSP rating scale. We found that worse motor performance (UPDRS-3) was associated with increased MD in the whole thalamus, VAVL, and LPVP (Spearman's Rho $=0.714$ $0.772, P<0.05)$ and reduced FA in the whole thalamus

b

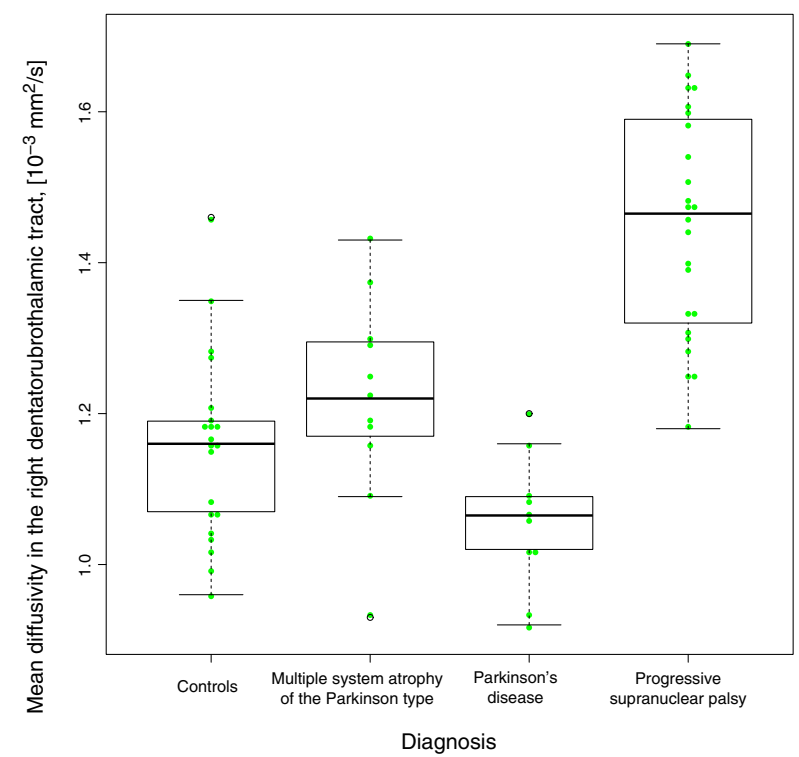

d

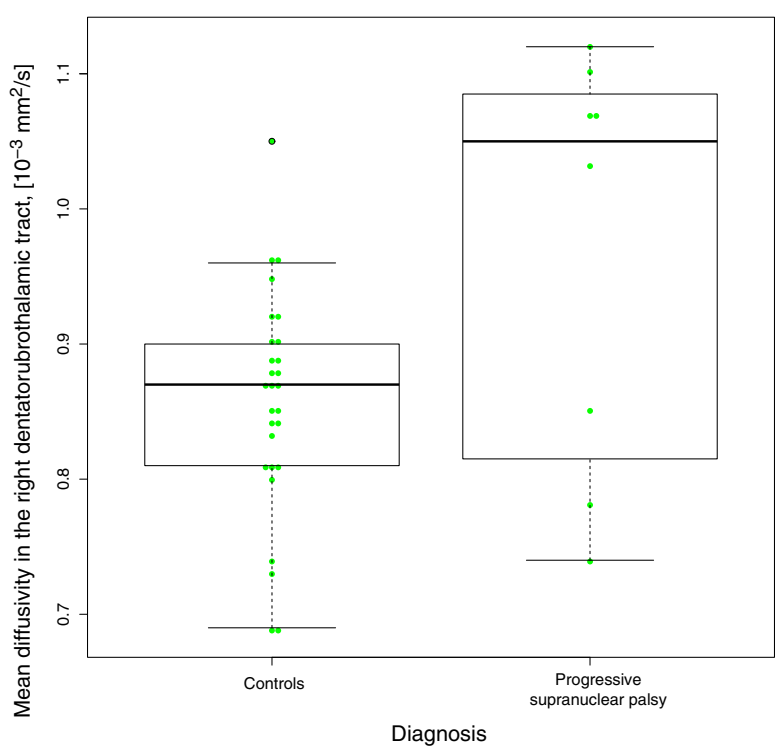

$3 \mathrm{~mm}^{\wedge} 2 / \mathrm{s}$ ]. c Fractional anisotropy values in the right dentatorubrothalamic tract derivation cohort. $\mathbf{d}$ Mean diffusivity values in the right dentatorubrothalamic tract derivation cohort, $\left[10^{\wedge}-3 \mathrm{~mm}^{\wedge} 2 / \mathrm{s}\right]$ 
(Spearman's Rho $=-0.762, P=0.028$ ). Impaired balance (Tandem Gait Test) correlated with increased MD in the whole thalamus, VAVL, LPVP, but also with MD in the right DRTT (Spearman's Rho $=0.791-0.828, P<0.05$ ). Further, we found significant correlations between diffusion changes in thalamus (whole thalamus and VAVL) and the items in the PSP rating scale (Table 3). As depicted in Fig. 4, linear regression showed highly significant positive linear correlation between MD in the thalamus in patients with PSP and the PSP rating scale total score and strong negative linear relationship between FA in the thalamus in PSP and the PSP rating scale total score.

\section{Diagnostic accuracy of diffusion parameters of the midbrain, dentatorubrothalamic tract, and thalamus in progressive supranuclear palsy}

Diffusion parameters from the validation cohort, that showed the most extensive differences between PSP and other diagnostic groups, were tested separately in the ROC analysis for diagnostic accuracy (Table 4).

When using the MD in the midbrain, we found that it could differentiate PSP from controls with an area under the curve (AUC) of 0.86 (Table 4), PSP from MSA-P and PD with an AUC of 0.90 (Table 4), and PSP from all groups of 0.88 (Table 4).

When using the MD in the right DRTT, we found that it could differentiate PSP from controls with an AUC of 0.95 (Table 4). Similar results were obtained when PSP separated from MSA-P and PD (Table 4) and when PSP separated from all groups (Table 4).

When using MD in the thalamus, we found that it could differentiate PSP from controls with an AUC of 0.77 (Table 4). Similar results were obtained when PSP separated from MSA-P and PD (Table 4) and when PSP separated from all groups (Table 4).

\section{Discussion}

We have performed a study comprising two cohorts with similar demographic characteristics, comparing DTI and volumetric measurements. We derived and validated changes of MD in the thalamus, in the VAVL and LVLP thalamic nuclei and in the DRTT in PSP. Importantly, these changes were specific for PSP and correlated highly with the PSP rating scale. Furthermore, these changes were not correlated with the atrophy of thalamus and seem associated with worse motor symptoms and impaired balance.

Neurodegeneration of the thalamic nuclei in PSP has been described in a few small autopsy studies [6, 43, 44]. Taucontaining neurofilaments (NFTs) have been shown in many nuclei of the thalamus in patients with PSP. In some cases, the loss of nerve cells was severe in the dorsal part of lateral and ventral nuclei with concomitant fibrillary gliosis. Further, a comparative neuropathological study comparing cases with PD and PSP found tau-pathology in all ventral thalamic nuclei in PSP with neuronal loss most evident in the ventrolateral posterior nuclei [45]. In vivo, DTI changes in PSP have previously been shown in the thalamus when compared to controls $[12,14,17]$. Our results corroborate these findings and further suggest that changes of MD in the thalamus, in the VAVL, and LVLP thalamic nuclei are specific for PSP when compared to the other major parkinsonian disorders such as PD and MSA-P.

The present findings of changes of DTI parameters in the SCP, midbrain, and ventral thalamic nuclei in PSP suggested structural damage along the DRTT. The DRTT projects from the dentate nucleus of the cerebellum, through the SCP toward the red nucleus (with axon collaterals to this nucleus) and then proceeds superiorly to the contralateral ventral lateral and anterior nuclei of the thalamus. Degeneration of DRTT has previously been shown neuropathologically in 10 cases with PSP where degeneration and activated microglia along this tract
Table 3 Spearman's correlation coefficient (Rho) describing the association of diffusion tensor imaging parameters mean diffusivity and fractional anisotropy with the Progressive Supranuclear Palsy Rating Scale items scores

\begin{tabular}{|c|c|c|c|c|c|}
\hline \multirow[t]{2}{*}{ PSPRS items } & \multirow[t]{2}{*}{ PSPRS items scores } & \multicolumn{2}{|l|}{ FA } & \multicolumn{2}{|l|}{$\mathrm{MD}$} \\
\hline & & Thalamus & VAVL & Thalamus & VAVL \\
\hline History & $7.25 \pm 4.62$ & -0.383 & -0.575 & 0.611 & 0.659 \\
\hline Mentation & $4.25 \pm 3.88$ & $-0.849 \ddagger$ & -0.506 & $0.892 \ddagger$ & $0.892 \ddagger$ \\
\hline Bulbar & $2 \pm 1.6$ & $-0.835 \dagger$ & $-0.933 末$ & 0.651 & $0.749 \dagger$ \\
\hline Ocular motor & $8.88 \pm 2.85$ & $-0.759 \dagger$ & -0.313 & 0.699 & $0.723 \dagger$ \\
\hline Limb motor & $5.75 \pm 1.67$ & $-0.776 \dagger$ & -0.325 & $0.801 \dagger$ & $0.776 \dagger$ \\
\hline Gate and midline & $11 \pm 7.19$ & $-0.735 \dagger$ & -0.578 & $0.783 \dagger$ & $0.807 \dagger$ \\
\hline Total & $39.13 \pm 18.11$ & $-0.833 \dagger$ & $-0.738 \dagger$ & $0.905 \$$ & $0.929 \ddagger$ \\
\hline
\end{tabular}

PSPRS items scores expressed as mean \pm standard deviation. No correction for multiple comparisons was done Abbreviations: PSPRS Progressive Supranuclear Palsy Rating Scale, MD mean diffusivity, FA fractional anisotropy, $V A V L$ ventral anterior and ventral lateral nuclei of thalamus

$\dagger P<0.05, \ddagger P<0.01$, Spearman's correlation coefficient 
Fig. 4 Correlation between the progressive supranuclear palsy rating score and fractional anisotropy and mean diffusivity in the thalamus for the patients with progressive supranuclear palsy in derivation cohort. a The regression line (black line) with a $95 \%$ confidence interval $(\mathrm{CI})$ shows an association between higher progressive supranuclear palsy rating score (PSPRS) and reduced fractional anisotropy in the thalamus. The correlation coefficient value is 0.81 and $60 \%$ of variance is explained. $\mathbf{b}$ The regression line (black line) with a $95 \%$ CI shows an association between higher PSPRS score and elevated mean diffusivity in the thalamus. The correlation coefficient value is 0.84 and $65 \%$ of variance is explained

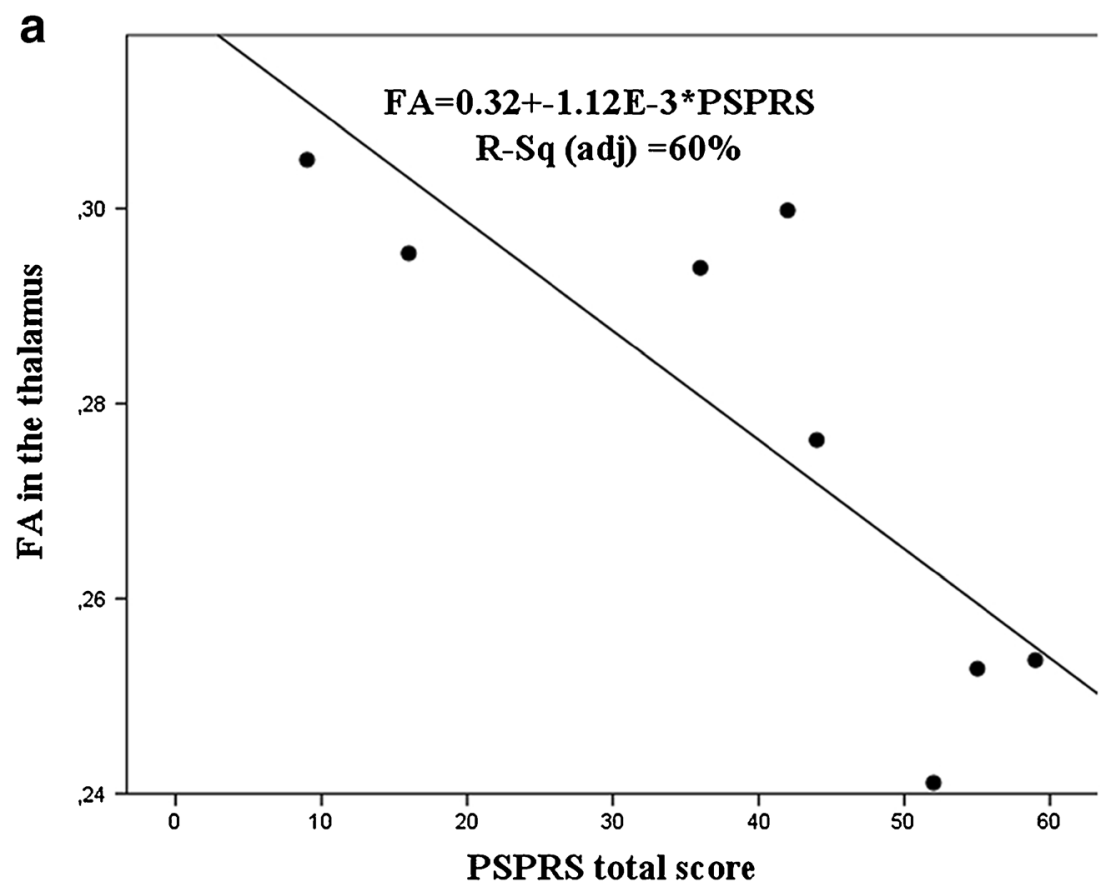

b

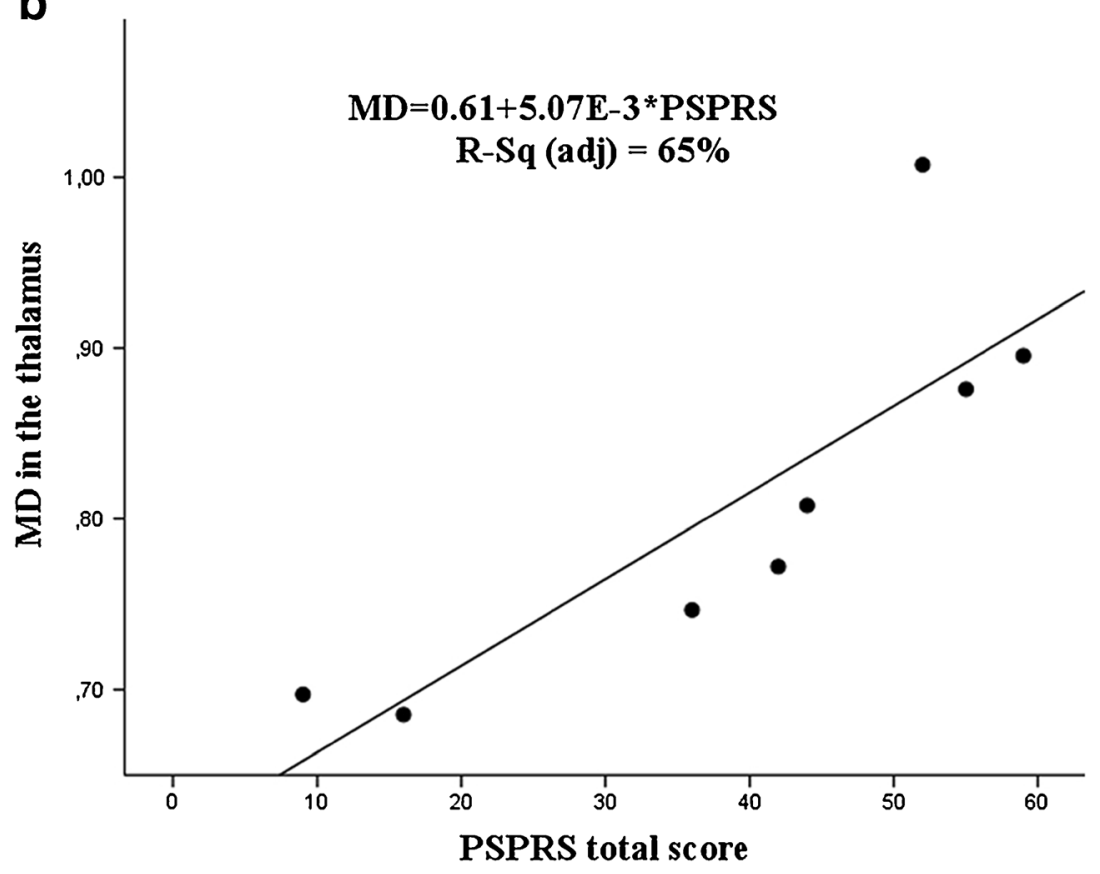

were found [46]. Using tractography, we demonstrate reduced FA and elevated MD in the DRTT in PSP, which was confirmed in two different cohorts (Table 2; Fig. 3). Further, the quite high diagnostic accuracy obtained using the MD in the DRTT and $\mathrm{MD}$ in the midbrain, and to a lesser degree the MD of thalamus (Table 4), warrants further studies investigating the clinical diagnostic utility of these measures. To our knowledge, this study was first to using tractography to show changes in diffusion parameters along the DRTT in patients with PSP but not the first to show diffusion changes in regional parts of the DRTT [14].
The motor and pre-motor cortex receives thalamic inputs especially from the ventral thalamic nuclei (i.e., the "motor thalamus") and changes in motor cortical activation are associated with the clinical features of rigidity, bradykinesia, and postural instability in both PSP and PD [45]. In this context, it is interesting to note that we found that increased MD in ventral thalamic nuclei was very consistently associated with worse motor symptoms in PSP. The ventral thalamic nuclei receive input, e.g., from the basal ganglia (globus pallidus) via the thalamic fasciculus and from the cerebellum via the DRTT. 
Table 4 Diagnostic accuracy of mean diffusivity in the thalamus, the right dentatorubrothalamic tract, and midbrain

\begin{tabular}{|c|c|c|c|c|}
\hline & ROC (95 \% CI) & Cutoff MD $\left[\mu \mathrm{m}^{2} / \mathrm{ms}\right]$ & Sensitivity $\%$ & Specificity $\%$ \\
\hline \multicolumn{5}{|c|}{ PSP vs controls } \\
\hline Thalamus & $0.77(0.64-0.91)$ & 0.80 & 74 & 67 \\
\hline DRTT R & $0.95(0.88-1.00)$ & 1.23 & 96 & 81 \\
\hline Midbrain & $0.86(0.74-0.98)$ & 0.77 & 85 & 81 \\
\hline \multicolumn{5}{|c|}{ PSP vs PD and MSA-P } \\
\hline Thalamus & $0.81(0.68-0.93)$ & 0.79 & 81 & 77 \\
\hline DRTT R & $0.94(0.87-1.0)$ & 1.25 & 92 & 81 \\
\hline Midbrain & $0.90(0.88-0.99)$ & 0.78 & 81 & 81 \\
\hline \multicolumn{5}{|c|}{ PSP vs PD, MSA-P, and controls } \\
\hline Thalamus & $0.79(0.68-0.9)$ & 0.8 & 74 & 71 \\
\hline DRTT R & $0.94(0.89-0.99)$ & 1.24 & 96 & 79 \\
\hline Midbrain & $0.88(0.79-0.97)$ & 0.77 & 85 & 79 \\
\hline
\end{tabular}

Values refer to the validation cohort

Abbreviations: PSP progressive supranuclear palsy, $P D$ Parkinson's disease, MSA-P multiple system atrophy, parkinsonian variant, $D R T T R$ right dentatorubrothalamic tract, $R O C$ receiver operator curve analysis, $M D$ mean diffusivity, $C I$ confidence interval

It has been suggested that the DRTT is important for postural stability. Our finding that increased MD in the DRTT is associated with impaired balance (poor performance on the tandem gait test) in patients with PSP might suggest that these changes are associated with postural instability and falls in PSP. This finding is in agreement with a previous study showing that imbalance and falls in PSP are associated with thalamic dysfunction visualized with FDGPET, suggesting that brainstem-thalamic loops play an important role in postural imbalance and falls in PSP [9].

In the present study, we also found that the volumes of the putamen and the globus pallidus are reduced in both MSA-P and PSP, but that the volumes of the thalamus and the midbrain are selectively reduced in PSP, which is in agreement with a previous study [47]. The volumetric changes of the thalamus were not associated with clinical assessment scales in PSP, which was in sharp contrast to diffusion measures (especially MD) in the same structure, indicating that DTI more reliably detect meaningful changes in the thalamus of patients with PSP.

Our DTI results of ROI-based analysis of diffusion changes in thalamus are in agreement with the TBSS analysis of thalamus. Interestingly, our DTI results of MD changes in the infratentorial part of the DRTT in patients with PSP are in agreement with previously published results of the TBSS study [48] where patients with PSP showed white matter (WM) changes encompassing the inferior part of this tract. Our results are also in line with a previous report from another TBSS study [49] showing widespread changes in white matter tracts in both PSP and MSA patients, not found in patients with PD.

Our study has some limitations. First, the PSP group is small in the derivation cohort. However, there are previous studies demonstrating that with effect sizes above $5 \%$, as few as 4-7 individuals are needed when analyzing MD values in white matter structures [50]. Although the coefficients of variation were slightly higher in the structures we analyzed, the reduction in MD in PSP compared to controls in derivation cohort was up to $15 \%$. Given that the results were reproduced in the validation cohort, we are confident in the reliability of the results. Second, when using small cohort number, the main limitation is the risk of type II errors, not type I errors. The strength of the present study was also that the results from the derivation cohort were reproduced in the validation cohort. However, the correlations performed only in the derivation cohort (i.e., correlations with UPDRS, PSP rating scale, and tandem gate) should be interpreted with caution until validated in other cohorts. Third, manual placements of ROIs for DTI analyses can be subjective, but the ROI-based analyses of the present study resulted in high intra-rater reliability and the results were validated by the automatic TBSS analysis. Finally, clinical diagnostic criteria were used for patient collection without neuropathological confirmation in the study, thus misdiagnosis cannot be excluded. However, in two cases (one with PSP and one with MSA-P) that underwent neuropathological examination the clinical diagnoses were confirmed.

\section{Conclusions}

We investigated disease-specific structural changes in thalamus and dentatorubrothalamic tract in PSP. In a cohort with 8 PSP patients and 30 controls, we found elevated MD in the thalamus, SCP, midbrain, and of the DRTT in patients with PSP. Increased MD in the thalamus and in the DRTT 
correlated with impaired motor function or balance in patients with PSP. Volumetric analysis showed reduced thalamic volumes in PSP. The DTI and volumetric findings were successfully reproduced in a validation cohort with 27 PSP patients and 21 controls. In addition, we found that most of these changes were specific to PSP and not found in patients with PD or MSA-P, which indicates that MD changes in thalamus and DRTT might be specific to impaired motor function and balance. Future studies need to be performed to examine whether changes in DTI parameters in the thalamus could be part of an MRI protocol for differential diagnosis of PSP vs PD and MSA-P. Further studies are also needed to confirm that alterations in thalamus and the DRTT are associated with impaired motor function and balance in PSP.

Acknowledgments The authors thank Ann Johansson and Katarina Johansson, Research Nurses in the Neurology Department, without whom completion of this study would never have been possible. The study was supported by the European Research Council, the Swedish Research Council, The Parkinson Foundation of Sweden, The Michael J Fox Foundation, the Crafoord Foundation, the Swedish Brain Foundation and the Swedish Federal Government under the ALF agreement. The funding sources had no role in the design and conduct of the study, in the collection, analysis, interpretation of the data or in the preparation, review or approval of the manuscript.

Ethical standards and patient consent We declare that all human and animal studies have been approved by the Ethics Committee at Lund University and have therefore been performed in accordance with the ethical standards laid down in the 1964 Declaration of Helsinki and its later amendments. We declare that all patients gave informed consent prior to inclusion in this study.

Conflict of interest We declare that we have no conflict of interest.

Open Access This article is distributed under the terms of the Creative Commons Attribution 4.0 International License (http:// creativecommons.org/licenses/by/4.0/), which permits unrestricted use, distribution, and reproduction in any medium, provided you give appropriate credit to the original author(s) and the source, provide a link to the Creative Commons license, and indicate if changes were made.

\section{References}

1. Steele JC, Richardson JC, Olszewski J (1964) Progressive supranuclear palsy. A heterogeneous degeneration involving the brainstem, basal ganglia and cerebellum with vertical gaze and pseudobulbar palsy, nuchal dystonia and dementia. Arch Neurol 10:333-59

2. Dickson DW, Ahmed Z, Algom AA et al (2010) Neuropathology of variants of progressive supranuclear palsy. Curr Opin Neurol 23: $394-400$

3. Litvan I, Agid Y, Calne D et al (1996) Clinical research criteria for the diagnosis of progressive supranuclear palsy (Steele-RichardsonOlszewski syndrome): report of the NINDS-PSP International Workshop. Neurology 47:1-9

4. Wakabayashi K, Takahashi H (2004) Pathological heterogeneity in progressive supranuclear palsy and corticobasal degeneration. Neuropathology 24:79-86
5. Williams DR, Lees AJ (2009) Progressive supranuclear palsy: clinicopathological concepts and diagnostic challenges. Lancet Neurol 8:270-79

6. Dickson DW, Rademakers R, Hutton ML (2007) Progressive supranuclear palsy: pathology and genetics. Brain Pathol 17:74-82

7. Srulijes K, Reimold M, Liscic RM et al (2012) Fluorodeoxyglucose positron emission tomography in Richardson's syndrome and progressive supranuclear palsy-parkinsonism. Mov Disord 27:151-5

8. Zalewski N, Botha H, Whitwell JL et al (2014) FDG-PET in pathologically confirmed spontaneous $4 \mathrm{R}$-tauopathy variants. J Neurol 261:710-6

9. Zwergal A, la Fougère C, Lorenzl S et al (2011) Postural imbalance and falls in PSP correlate with functional pathology of the thalamus. Neurology 77:101-9

10. Basser PJ, Mattiello J, LeBihan D (1994) MR diffusion tensor spectroscopy and imaging. Biophys J 66:259-67

11. Hess CP, Christine CW, Apple AC et al (2014) Changes in the thalamus in atypical parkinsonism detected using shape analysis and diffusion tensor imaging. AJNR Am J Neuroradiol 35:897-903

12. Saini J, Bagapally BS, Sandhya M et al (2012) In vivo evaluation of white matter pathology in patients of progressive supranuclear palsy using TBSS. Neuroradiology 54:771-80

13. Wang J, Wai Y, Lin WY et al (2010) Microstructural changes in patients with progressive supranuclear palsy: a diffusion tensor imaging study. J Magn Reson Imaging 32:69-75

14. Whitwell JL, Avula R, Master A et al (2011) Disrupted thalamocortical connectivity in PSP: a resting-state fMRI, DTI, and VBM study. Parkinsonism Relat Disord 17:599-605

15. Blain CR, Barker GJ, Jarosz JM et al (2006) Measuring brain stem and cerebellar damage in parkinsonian syndromes using diffusion tensor MRI. Neurology 67:2199-205

16. Nilsson C, Markenroth Bloch K, Brockstedt S et al (2007) Tracking the neurodegeneration of parkinsonian disorders - a pilot study. Neuroradiology 49:111-9

17. Erbetta A, Mandelli ML, Savoiardo M et al (2009) Diffusion tensor imaging shows different topographic involvement of the thalamus in progressive supranuclear palsy and corticobasal degeneration. AJNR Am J Neuroradiol 30:1482-7

18. Kwon HG, Hong JH, Hong CP et al (2011) Dentatorubrothalamic tract in human brain: diffusion tensor tractography study. Neuroradiology 53:787-91

19. Hoehn MM, Yahr MD (1967) Parkinsonism: onset, progression and mortality. Neurology 17:427-442

20. Schwab RS, England AC (1969) Projection technique for evaluating surgery in Parkinson's Disease. In: Gillingham FJ, Donaldson IML (eds) Third symposium of Parkinson's disease. Edinburgh, Scotland, pp 152-57

21. Fahn S, Elton RL, members of the UPDRS development committee (1987) Unified Parkinson's Disease Rating Scale. In: Fahn S, Marsden CD, Goldstein M, Calne DB (eds) Recent developments in Parkinson's Disease, vol 2. Macmillan Healthcare Information, Florham Park, NJ, pp 153-63

22. Fregly AR (1974) Vestibular ataxia and its measurement in man. In: Kornhuber HH (ed) Handbook of sensory physiology. Springer, Berlin, pp 321-60

23. Folstein SE, McHugh PR (1975) "Mini-mental state": a practical method for grading the cognitive state of patients for the clinician. J Psychiatr Res 12:189-98

24. Nielsen NP, Wiig EH, Warkentin S et al (2004) Clinical utility of color-form naming in Alzheimer's disease: preliminary evidence. Percept Mot Skills 99:1201-4

25. Golbe LI, Ohman-Strickland PA (2007) A clinical rating scale for progressive supranuclear palsy. Brain 130:1552-65

26. Calne DB, Snow BJ, Lee C (1992) Criteria for diagnosing Parkinson's disease. Ann Neurol 32:S125-7 
27. Gilman S, Wenning GK, Low PA et al (2008) Second consensus statement on the diagnosis of multiple system atrophy. Neurology 71:670-676

28. Nucifora PG, Verma R, Lee SK, Melhem ER (2007) Diffusiontensor MR imaging and tractography: exploring brain microstructure and connectivity. Radiology 245:367-84

29. Klein S, Staring M, Murphy K et al (2010) Elastix: a toolbox for intensity-based medical image registration. IEEE Trans Med Imaging 29:196-205

30. Tournier JD, Calamante F, Connelly A (2007) Robust determination of the fibre orientation distribution in diffusion MRI: nonnegativity constrained super-resolved spherical deconvolution. Neuroimage 35:1459-72

31. Wiegell MR, Tuch DS, Larsson HB et al (2003) Automatic segmentation of thalamic nuclei from diffusion tensor magnetic resonance imaging. Neuroimage 19:391-401

32. Unrath A, Klose U, Grodd W et al (2008) Directional colour encoding of the human thalamus by diffusion tensor imaging. NeurosciLett 434:322-7

33. Oba H, Yagishita A, Terada $\mathrm{H}$ et al (2005) New and reliable MRI diagnosis for progressive supranuclear palsy. Neurology 64:2050 55

34. Smith SM, Jenkinson M, Johansen-Berg H et al (2006) Tract-based spatial statistics: voxelwise analysis of multi-subject diffusion data. NeuroImage 31:1487-1505

35. Andersson JLR, Jenkinson M, Smith S (2012) Non-linear optimisation. FMRIB Technical Report TR07JA1 2007a http:// wwwfmriboxacuk/analysis/techrep Accessed(3 September 2012)

36. Andersson JLR, Jenkinson M, Smith S (2012) Non-linear registration, aka Spatial normalisation. FMRIB Technical Report TR07JA2. 2007b. http://www.fmrib.ox.ac.uk/analysis/techrep Accessed 3 September 2012

37. Smith SM (2002) Fast robust automated brain extraction. Human Brain Mapping 17:143-155

38. Smith SM, Jenkinson M, Woolrich MW et al (2004) Advances in functional and structural MR image analysis and implementation as FSL. Neuroimage 23:S208-S219

39. Quattrone A, Nicoletti G, Messina D et al (2008) MR imaging index for differentiation of progressive supranuclear palsy from
Parkinson disease and the Parkinson variant of multiple system atrophy. Radiology 246:214-21

40. Patenaude B, Smith SM, Kennedy D et al (2011) A Bayesian model of shape and appearance for subcortical brain. NeuroImage 56:90722

41. Buckner RL, Head D, Parker J et al (2004) A unified approach for morphometric and functional data analysis in young, old, and demented adults using automated atlas-based head size normalization: reliability and validation against manual measurement of total intracranial volume. Neuroimage 23:724-38

42. Winkler AM, Ridgway GR, Webster MA et al (2014) Permutation inference for the general linear model. NeuroImage 92:381-397

43. Henderson JM, Carpenter K, Cartwright $\mathrm{H}$ et al (2000) Loss of thalamic intralaminar nuclei in progressive supranuclear palsy and Parkinson's disease: clinical and therapeutic implications. Brain 123:1410-21

44. Kobayashi Z, Akaza M, Ishihara S et al (2013) Thalamic hypoperfusion in early stage of progressive supranuclear palsy (Richardson's syndrome): Report of an autopsy-confirmed case. J Neurol Sci 335:224-7

45. Halliday GM, Macdonald V, Henderson JM (2005) A comparison of degeneration in motor thalamus and cortex between progressive supranuclear palsy and Parkinson's disease. Brain 128:2272-80

46. Ishizawa K, Dickson DW (2001) Microglial activation parallels system degeneration in progressive supranuclear palsy and corticobasal degeneration. J Neuropathol Exp Neurol 60:647-57

47. Messina D, Cerasa A, Condino F et al (2011) Patterns of brain atrophy in Parkinson's disease, progressive supranuclear palsy and multiple system atrophy. Parkinsonism and Related Disorders 17:172-6

48. Tessitore A, Giordano A, Caiazzo G et al (2014) Clinical correlations of microstructural changes in progressive supranuclear palsy. Neurobiol Aging 35:2404-10

49. Worker A, Blain C, Jarosz J et al (2014) Diffusion tensor imaging of Parkinson's disease, multiple system atrophy and progressive supranuclear palsy: a tract-based spatial statistics study. PLoS One 9(11), e112638

50. Heiervang E, Behrens TEJ, Mackay CE et al (2006) Between session reproducibility and between subject variability of diffusion MR and tractography measures. NeuroImage 33:867-877 